
Informations

une revue Gallia

Rhône-Alpes | 2009

\title{
Valence
}

Musée des Beaux Arts et d'Archéologie

\section{Pascale Réthoré}

\section{(2) OpenEdition \\ 12 Journals}

Édition électronique

URL : http://journals.openedition.org/adlfi/3434

ISSN : 2114-0502

Éditeur

Ministère de la culture

Référence électronique

Pascale Réthoré, «Valence », ADLFI. Archéologie de la France - Informations [En ligne], Rhône-Alpes, mis en ligne le 01 mars 2009, consulté le 19 avril 2019. URL : http://journals.openedition.org/adlfi/3434

Ce document a été généré automatiquement le 19 avril 2019

(c) Ministère de la Culture et de la Communication, CNRS 


\title{
Valence
}

\author{
Musée des Beaux Arts et d'Archéologie
}

\section{Pascale Réthoré}

\section{Identifiant de l'opération archéologique : 10160}

Date de l'opération : 2009 (SP)

L'ancien palais épiscopal-musée est un site patrimonial majeur à forte sensibilité archéologique. Sa rénovation-extension par la Ville de Valence a conduit l'Etat à prescrire une fouille archéologique dans les secteurs appelés à être transformés ou rénovés. Cette intervention est répartie en trois phases:avant (2009) et après démolitions (2010), pendant les travaux (2010-2013).

La réfection de la place des Ormeaux en 2003 avait été l'occasion d'identifier le premier quartier résidentiel de l'évêque succédant, à partir des $\mathrm{IV}^{\mathrm{e}} \mathrm{s}$.- $\mathrm{V}^{\mathrm{e}} \mathrm{s}$. à un habitat du début de notre ère. Parallèlement, une étude d'archéologie du bâti révélait à l'emplacement du corps principal du musée, le coeur du palais épiscopal construit au XII ${ }^{\mathrm{e}} \mathrm{s}$. La résidence primitive des évêques s'était donc déplacée vers l'ouest en rebord de terrasse, entre le haut et le bas Moyen Âge.

Les fouilles de 2009 confirment cette évolution et la précisent. En revanche, l'occupation antique dans ce secteur apparaît bouleversée. L'étude des données de fouilles, actuellement en cours, ne verra ses conclusions aboutir qu'après la dernière phase de fouille de l'automne 2010. Un premier plan par grandes phases chronologiques a été établi. (Fig. $n^{\circ} 1$ : Plan général de la fouille avec grands phasages archéologiques : zone 1 : sondage dans le jardin, zone 2: ancienne salle d'exposition archéologique, zone 3 : ancienne salle d'exposition des sculptures, zone 4 : cour d'honneur.)

\section{Un quartier antique à caractère monumental ?}

L'habitat antique, reconnu sous la place des Ormeaux, de construction modeste, n'a pas été retrouvé à l'emplacement de l'actuel musée. Pourtant, deux murs parallèles nord-sud, 
avec leurs seuils et un retour est-ouest pourraient lui être associés. Repris au III ${ }^{\mathrm{e}}$ s., leur origine pourrait être plus ancienne. L'espace de $11 \mathrm{~m}$ de large entre ces deux murs fait penser à une rue en accord avec le réseau viaire actuellement connu pour la ville antique. Mais l'ensemble du terrain a été si perturbé par de vastes excavations au Bas-Empire et au haut Moyen Âge que les niveaux les plus anciens ne sont plus en place et cette proposition d'interprétation doit être prise avec réserve.

$\mathrm{Au}$ sud-est de cet axe, plusieurs murs de belle construction, larges de 0,88 m, dont l'un forme une abside potentielle de $12 \mathrm{~m}$ de diamètre, sont datés du début de notre ère et se distinguent par leur caractère monumental.

Un mur de 1,40 m de large construit en petits moellons de grès, observé en deux segments dans le jardin et la cour d'honneur du musée borne le côté ouest de cet ensemble imposant. Ce mur s'arrête à la hauteur d'un mur de blocs de molasse en grand appareil observé en 2003 au sud de la place des Ormeaux et daté du III ${ }^{\mathrm{e}}$ s., avec lequel il pourrait faire retour.

Le quartier antique de la place des Ormeaux semble donc voisiner avec un bâtiment monumental établi dès l'origine à l'angle sud-ouest de la cité, mais fortement réaménagé à partir du III $\mathrm{s}$. La fouille sous l'ancienne Conservation désormais détruite, devrait apporter de nouvelles données sur ce bâtiment.

\section{Évolution du palais épiscopal du haut Moyen Âge au Moyen Âge classique}

Un four à chaux de $4 \mathrm{~m}$ de diamètre, associé à une aire de travail est sans doute à mettre en rapport avec la construction du premier palais épiscopal. Sa présence traduit, la proximité d'un bâti imposant à construire, et nécessitant donc une production de chaux directement sur le chantier. Cependant, la fouille sectorisée n'a pas permis de dégager un plan complet de la première maison de l'évêque.

Les vestiges observés dans la cour d'honneur ne sont pas à usage d'habitat. Deux murs de facture médiocre mais courant d'est en ouest sur toute la longueur de cette cour marquent vraisemblablement les limites successives d'espaces extérieurs, de type cours ou jardins. C'est peut-être également le rôle d'un vaste fossé, creusé au Moyen Âge, large de 4,5 $\mathrm{m}$ et profond de $1,60 \mathrm{~m}$, orienté lui aussi est-ouest.

A partir des $\mathrm{XI}^{\mathrm{e}}$ s.-XII ${ }^{\mathrm{e}}$ s., est construit plus à l'ouest, en bordure de terrasse, le mur de façade du bâtiment, en lien avec la tour primitive du palais médiéval. Lui est associé une vaste ère d'ensilage :vingt-six fosses silos (grenier à céréales) ont été repérées sur les zones fouillées. Elles représentent sans doute une infime part des réserves à grains du complexe épiscopal. Utilisées dans un second temps comme dépotoirs, elles ont livré une faune abondante et variée, traduisant le mode de vie aisé de la maison des évêques.

Quant à l'histoire du palais à la période moderne, c'est l'étude des murs décroutés de leurs enduits qui va prendre la suite de l'archéologie du sous-sol. Cette archéologie du bâti vient à peine de commencer. Néanmoins des sols en calade et quelques murs transversaux aujourd'hui détruits ont pu être mis au jour. Ils permettront de compléter l'analyse du bâti (Fig. n ${ }^{\circ} 2$ : Fouille des niveaux modernes dans l'ancienne salle de sculpture du musée (cliché Loïc de Cargouët). ). 


\section{ANNEXES}

Fig. $\mathrm{n}^{\circ} 1$ : Plan général de la fouille avec grands phasages archéologiques : zone $1:$ sondage dans le jardin, zone 2 : ancienne salle d'exposition archéologique, zone 3 : ancienne salle d'exposition des sculptures, zone 4 : cour d'honneur.

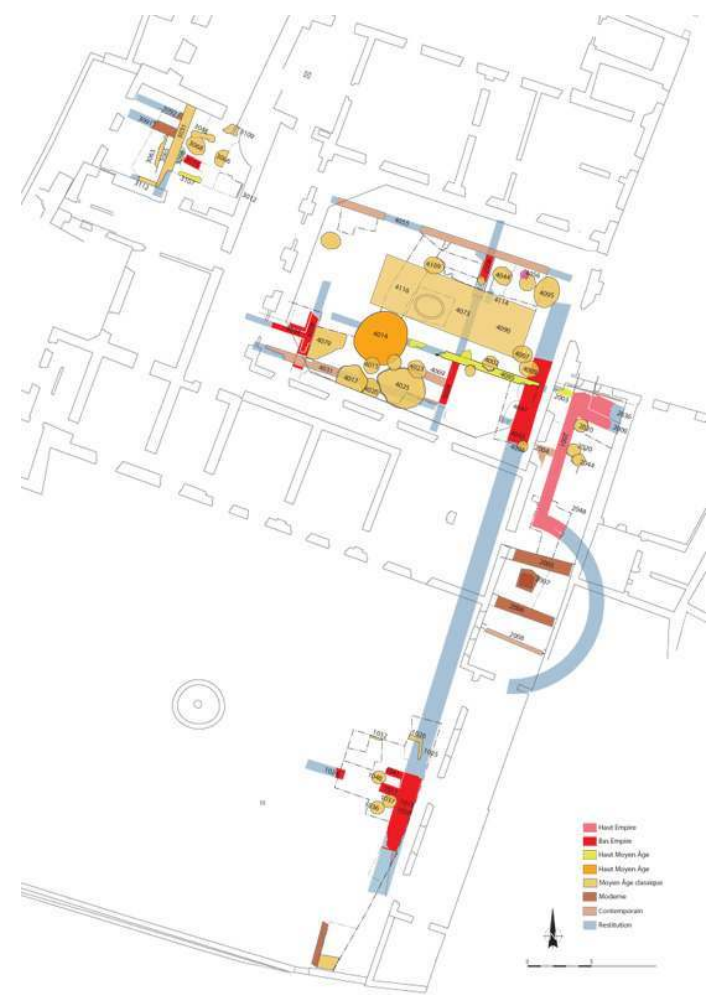

Auteur(s) : collectif (INRAP). Crédits : Collectif INRAP (2009) 
Fig. $\mathrm{n}^{\circ} 2$ : Fouille des niveaux modernes dans l'ancienne salle de sculpture du musée (cliché Loïc de Cargouët).

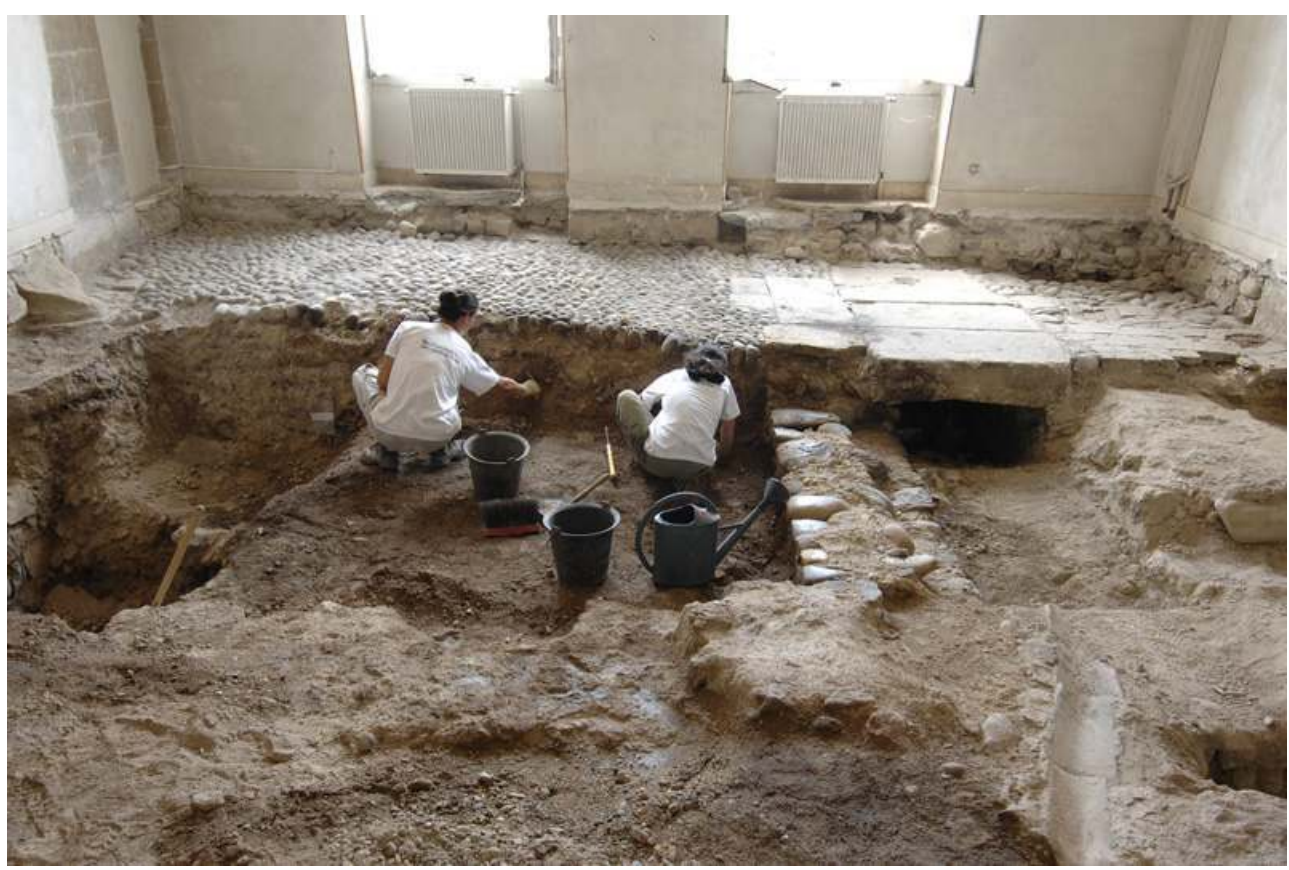

Auteur(s) : De Cargouët, Loïc (INRAP). Crédits : L.de Cargouët (2009)

\section{INDEX}

Index chronologique : IIIe siècle apr. J.-C., IVe siècle apr. J.-C., Ve siècle apr. J.-C., XIIe siècle apr. J.-C.

Index géographique : Rhône-Alpes, Drôme (26), Valence operation Sauvetage programmé (SP)

Thèmes : évêque, four à chaux, mur, palais épiscopal

\section{AUTEUR}

\section{PASCALE RÉTHORÉ}

INRAP 